\title{
The prevalence of myocardial bridging on multidetector computed tomography and its relation to coronary plaques
}

\author{
Ravindran Rajendran ${ }^{1 A, B, C, D, E, F}$, Madhav Hegde ${ }^{2 A, B, C, D, E, F}$ \\ 'Trichy SRM Medical College Hospital \& Research Centre, Irungalur, Trichy, Sri Jayadeva Institute of Cardiovascular Sciences and Research, Bangalore, India \\ ${ }^{2} \mathrm{BGS}$ Global Institute of Medical Sciences, Bangalore, India
}

\section{Abstract}

Purpose: To test the hypothesis that the prevalence of myocardial bridging varies between ethnic groups, and that the segment proximal to the myocardial bridge is more prone to plaque formation.

\begin{abstract}
Material and methods: A total of 4500 patients who had undergone computerised tomography (CT) coronary angiography at our institute were studied for myocardial bridging. Data on the clinical profile and indication for CT coronary angiography in myocardial bridging were collected. Patients with and without proximal disease were compared using the chi-square test for ordinal variables and Student's t-test for continuous variables. The length to depth ratio (RA-MA ratio) of the bridged segment was determined.
\end{abstract}

Results: The prevalence of atherosclerotic plaques in the segment proximal to the bridged segment was $37.8 \%$, which was lower than the prevalence of $48.7 \%$ for plaques in the corresponding segments among patients without myocardial bridging. The average length of the bridged segment was $15.5 \pm 5 \mathrm{~mm}$, and that for patients with and without proximal plaques was $13 \pm 4$ and $16 \pm 6 \mathrm{~mm}(p=0.1)$, respectively. Similarly, the average depth of the segments with and without proximal plaques was $1.8 \pm 0.6 \mathrm{~mm}$ and $1.4 \pm 0.5 \mathrm{~mm}(p=0.06)$, respectively. Only the RA-MA ratio ( $8 \pm 3$ vs. $13 \pm 6, p=0.01$ ) was significantly lower in patients with atherosclerotic plaques.

Conclusions: The prevalence in our study population was $10 \%$, with mid left anterior descending artery (LAD) being the most common segment involved. Moreover, the prevalence and distribution of coronary plaques in LAD were similar in patients with and without myocardial bridging.

Key words: CT coronary angiogram, myocardial bridging, coronary plaque, epidemiology, bridging ratio, CAD.

\section{Introduction}

Myocardial bridging is defined as an intramural course of an epicardial coronary artery for a variable length. The muscle overlying the tunnelled vessel is called the bridge. The autopsy description of myocardial bridging by Reyman [1] dates to 1732, whereas the first angiographic demonstration was provided by Portmann [2] in 1960. Initially, it was presumed to be benign with favourable long-term outcomes [3]. Subsequently, various clinical syndromes were proposed to be associated with myocardial bridging, including coronary spasm, acute coronary syndromes [4-6], ventricular arrhythmias, sudden cardiac death [7-10], and coronary atherosclerotic plaques proximal to the bridged segment [11]. The prevalence of myocardial bridging varies widely depending on the type of the study, and even the sex distribution varies considerably, with some studies showing either a male or a female predilection and the rest describing an equal prevalence in both sexes [11]. In autopsy series [12-14], the prevalence varies from $20 \%$ to $85 \%$, which is substantially higher than that in angiographic studies [15] (0.5-3\%); however, when patients with chest pain syndromes and normal coronary angiograms were studied using provocative tests,

Correspondence address:

Dr. Madhav Hegde, D 1003, The Gardens Apartments, Bangalore, Magadi Road, Bangalore, 560023, India, e-mail: docmadhavhegde@gmail.com

Authors' contribution:

A Study design · B Data collection · C Statistical analysis · D Data interpretation · E Manuscript preparation · F Literature search · G Funds collection 
the prevalence was as high as $40 \%$ on conventional angiograms $[16,17]$. With the advent of multislice computed tomography (CT), CT coronary angiography (CTCA), and cardiac magnetic resonance imaging, there has been a paradigm shift in the modality preferred for identifying coronary anomalies, including myocardial bridging; such identification is facilitated by the three-dimensional and multiplanar reformats available with these modalities. As the $\mathrm{CT}$ acquisition time is shortened with advanced equipment, motion artefacts are fewer, and myocardial bridging can be identified more accurately, but even with CTCA, the prevalence of myocardial bridging varies considerably from $3.5 \%$ to $58 \%[11,18-20]$. This wide variation can be attributed to different generations of CT equipment used in various studies; with quicker image acquisition requiring shorter breath holding times, artefacts and misinterpretations decrease considerably [21]. Another reason could be the ethnicity of the studied population, as observed in an autopsy study [11]. In the present study, we examined the CT prevalence of myocardial bridging and its association with coronary plaques in the Indian population at a high-volume tertiary care centre. Approval from the Institutional Ethics Committee was obtained.

\section{Material and methods}

\section{Study population}

A total of 4500 individuals who had undergone CTCA for evaluation of chest pain syndromes at our institute were studied for myocardial bridging. Moreover, 100 of them were excluded due to suboptimal imaging secondary to patient characteristics (obesity) and motion artefacts. Data on the clinical profile and indication for CTCA in patients with myocardial bridging were collected. An equal number of age-, sex-, and risk profile-matched controls without myocardial bridging were included in this study, and similar data were collected from them for comparison.

\section{Multidetector computed tomography protocol}

CTCA was conducted using 64-slice multidetector computed tomography (MDCT) after patients received $1 \mathrm{ml} /$ $\mathrm{kg}$ intravenous iohexol. The scanning area spanned from the tracheal carina to the diaphragm, with patients in the supine position. Only patients with sinus rhythm were included in this study, and the heart rate was reduced to $<80 / \mathrm{min}$ using oral metoprolol given one hour before the study. Additional IV metoprolol was used whenever required for better control of the heart rate. ECG-gated images were obtained during breath holding through detector collimation with the following parameters: detector collimation $-64 \times 0.625 \mathrm{~mm}$; voltage $120 \mathrm{kVp}$; effective mAs of 400-500 mAs; pitch range $0.2-0.29$; gantry rotation time -0.35 seconds; and slice thickness $-0.6 \mathrm{~mm}$.

\section{Myocardial bridging and coronary plaques}

The presence of myocardial bridging was assessed using transverse images and curved multiplanar reconstructed images (Figure 1) when a part of the epicardial coronary artery dipped within the myocardium only to return back to the surface. The total length from the point of deviation from the surface to the point of return to the surface was measured as the length of the bridged segment, and the thickness of the myocardium overlying the bridged segment was measured as the depth of the bridged segment (Figure 2). We assumed the length-to-depth ratio (RA-MA ratio) of the bridged segment as an indirect measure of angulation, with lower values reflecting a more acute angle and vice versa. A coronary plaque is any thickening of the vessel wall with or without narrowing of the lumen, and it is graded into normal, insignificant ( $<50 \%$ narrowing), and significant (> 50\% narrowing).

\section{Statistical analysis}

Descriptive statistics were calculated for the demographic profile. Patients with and without proximal disease were compared using the $\chi^{2}$ test for ordinal variables and Student's $t$-test for continuous variables. Similar tests were used for comparing patients with and without myocardial bridging.

\section{Results}

Of the 4400 patients included in the study, 440 had evidence of myocardial bridging, of whom 370 had bridging involving a major epicardial coronary artery, and the remaining patients had bridging involving smaller vessels. Among these 370 patients, 360 had bridging of the proximal to mid left anterior descending artery (LAD) segment, and the remaining 10 patients had bridging of the mid left circumflex artery (LCX). Seventy patients had bridging involving minor epicardial vessels (30 diagonals and 40 major obtuse marginals) that were still larger than $1.5 \mathrm{~mm}$ but were excluded from analysis because of proximal segment plaquing. $10.1 \%$ (160 of 1590) of women and 9.9\% (280 out of 2810 ) of men had myocardial bridging. As shown in Table 1, baseline characteristics were comparable between these 370 patients with major epicardial vessel myocardial bridging and their matched controls. Based on their age, sex, and type of chest pain, all these patients were evaluated for chest pain syndrome and intermediate pretest probability of coronary artery disease. The prevalence of any or significant atherosclerotic plaque (Figures 3 and 4) in the segment proximal to the bridged segment was $37.8 \%$, which was lower than the prevalence of $48.7 \%$ for plaques in corresponding segments among patients without myocardial bridging, who were matched for all other variables (Table 2). Moreover, the prevalence of plaques elsewhere in the coronary arteries was not dif- 

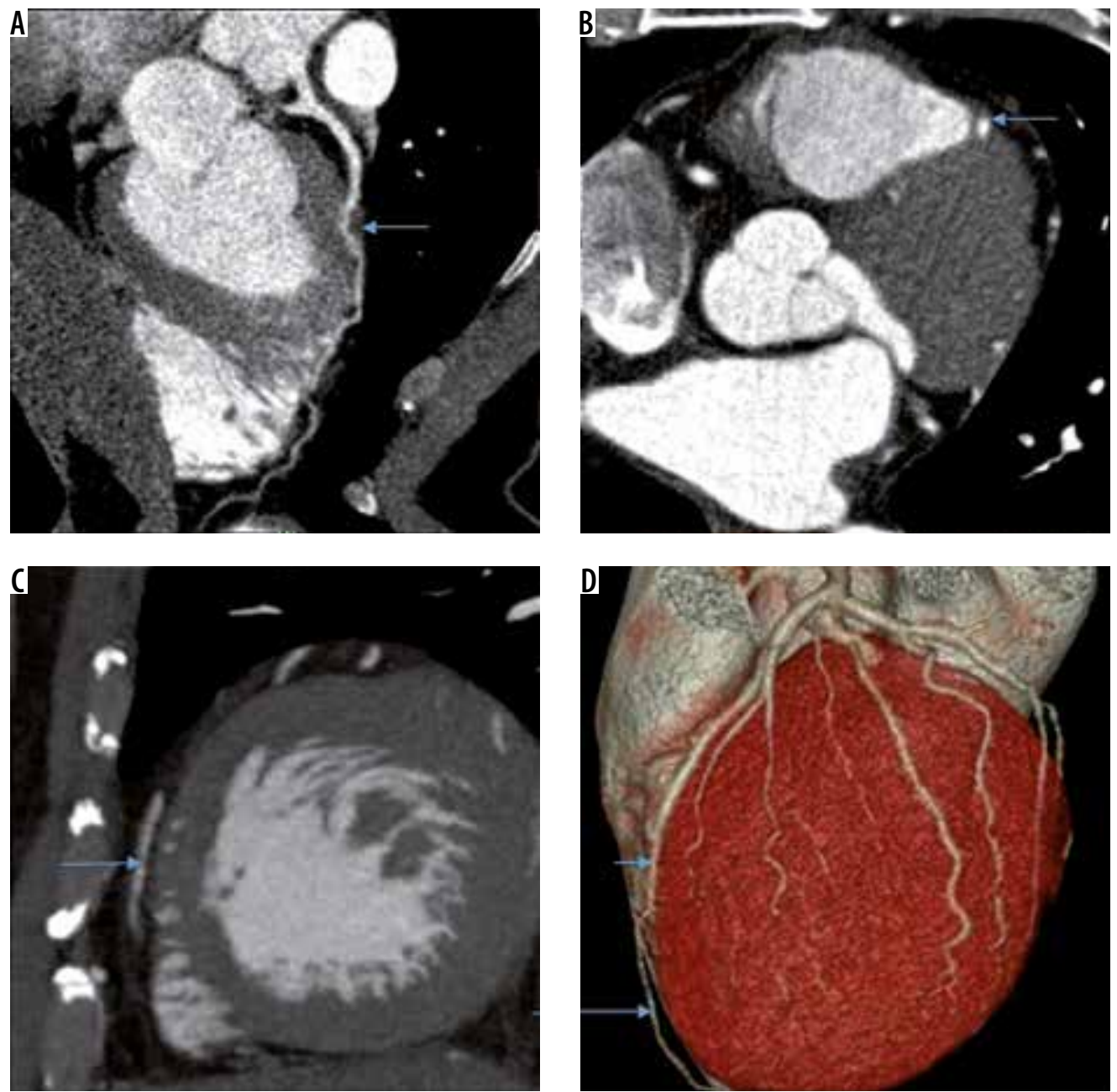

Figure 1. A) Curved multiplanar reformat image showing myocardial bridging of mid left anterior descending artery (LAD) (arrow). B) Transverse image showing myocardial bridging of LAD (arrow). C) Sagittal reformat showing normal epicardial course of distal LAD (arrow). D) Volume rendered image showing myocardial bridging of mid LAD (short arrow) and normal epicardial course of distal LAD (long arrow)

ferent between the two groups (patients with and without proximal plaques), reinforcing the appropriateness of matching. The average length of the bridged segment was $15.5 \pm 5 \mathrm{~mm}$, and that in patients with and without proximal plaques was $13 \pm 4$ and $16 \pm 6 \mathrm{~mm}(p=0.1)$, respectively. Similarly, the average depth of the segments with and without proximal plaques was $1.8 \pm 0.6 \mathrm{~mm}$ and $1.4 \pm$ $0.5 \mathrm{~mm}(p=0.06)$, respectively. No statistically significant differences were observed between the two groups, whereas the RA-MA ratio ( $8 \pm 3$ vs. $13 \pm 6, p=0.01$ ) was significantly lower in patients with atherosclerotic plaques. In univariate analysis, age and the presence of diabetes were also significantly associated with the presence of plaques proximal to the bridge among patients with myocardial bridging. In multivariate analysis, only age was found to be significant.

\section{Discussion}

In this study, the 64-slice MDCT prevalence of myocardial bridging among this cohort of Indian patients with chest

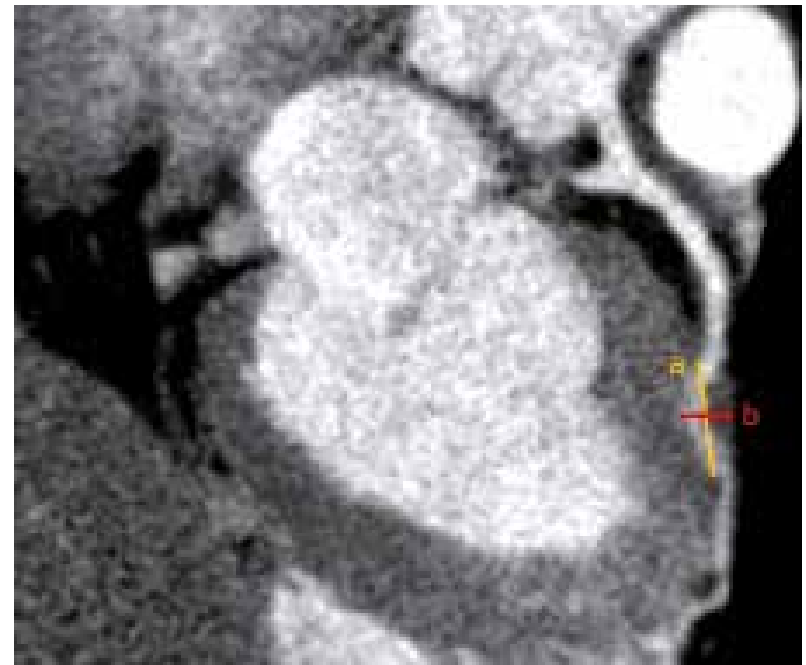

Figure 2. Orthogonal or oblique multiplanar reformatted image in which entry and exit of bridged segment is chosen. Using electronic callipers the distance (a) between the entry and exit point (outer to outer wall of the vessel) of the bridged segment was determined. The shortest distance (b) between epicardial surface and deeper myocardial aspect of the bridged segment was determined. The ratio was derived by using the formula of $\mathrm{a} / \mathrm{b}$ 
Table 1. Baseline characteristics

\begin{tabular}{|l|c|c|c|}
\hline Parameter & $\begin{array}{c}\text { Cases } \\
(n=370)\end{array}$ & $\begin{array}{c}\text { Controls } \\
(n=390)\end{array}$ & $p$ value \\
\hline Age (mean \pm SD) & $51 \pm 10$ & $52 \pm 11$ & 0.81 \\
\hline Females & $120(32.4 \%)$ & $160(41.6 \%)$ & 0.43 \\
\hline Diabetes & $100(27 \%)$ & $110(28.2 \%)$ & 0.56 \\
\hline Hypertension & $110(29.7 \%)$ & $110(28.2 \%)$ & 0.71 \\
\hline Smoking & $50(13.5 \%)$ & $50(12.8 \%)$ & 0.34 \\
\hline
\end{tabular}

Table 2. Prevalence of proximal segment plaque among cases and controls

\begin{tabular}{|l|c|c|c|c|}
\hline \multirow{2}{*}{} & \multicolumn{3}{|c|}{ Proximal vessel } & \multirow{2}{*}{ Total } \\
\cline { 2 - 4 } & Normal & $\begin{array}{c}\text { Insignificant } \\
\text { disease }\end{array}$ & $\begin{array}{c}\text { Significant } \\
\text { disease }\end{array}$ & \\
\hline Cases & $230(62.2 \%)$ & $80(21.6 \%)$ & $60(16.2 \%)$ & 370 \\
\hline Total & 430 & 210 & 120 & 760 \\
\hline
\end{tabular}

pain syndromes and intermediate pretest probability of CAD was $10 \%$, which is lower than the prevalence in other studies [22]. We presume the lower prevalence to be secondary to ethnicity because this is the first CT-based study to evaluate the prevalence among Indian patients. Moreover, we included only cases with definite evidence of bridging and excluded equivocal cases and those suggestive of motion artefacts. This led to higher specificity and lower sensitivity and hence lower prevalence. Finally, bridging involving vessels less than $1.5 \mathrm{~mm}$ in diameter was excluded because the mural course of these vessels may be considered normal even otherwise. Male and female patients were equally affected in our study, which is similar to the observation in other studies [11]. Similar to other studies, in this study, the most common vessel involved was $\mathrm{LAD}$, and mid-LAD was the most common segment $[22,23]$. Plaquing of the segment proximal to the bridged segment and the protection of the bridged segment were attributed to the shear stress on the endothelium caused by blood flow, which was considered lower than normal for the bridged segment and higher for the proximal segment. This shear stress can be presumed to be proportional to the amount of systolic deformation and its persistence in diastole, which in turn is dependent on the length, depth, RA-MA ratio, and myocardial contraction. We propose that a smaller RA-MA ratio indicates sharper angulation than a longer or a deeper segment alone can predict. In support of this, our study showed that the RA-MA ratio was significantly lower in patients with proximal plaquing than in patients without proximal plaquing, whereas the length or the depth of the bridged segment alone was not significantly different between
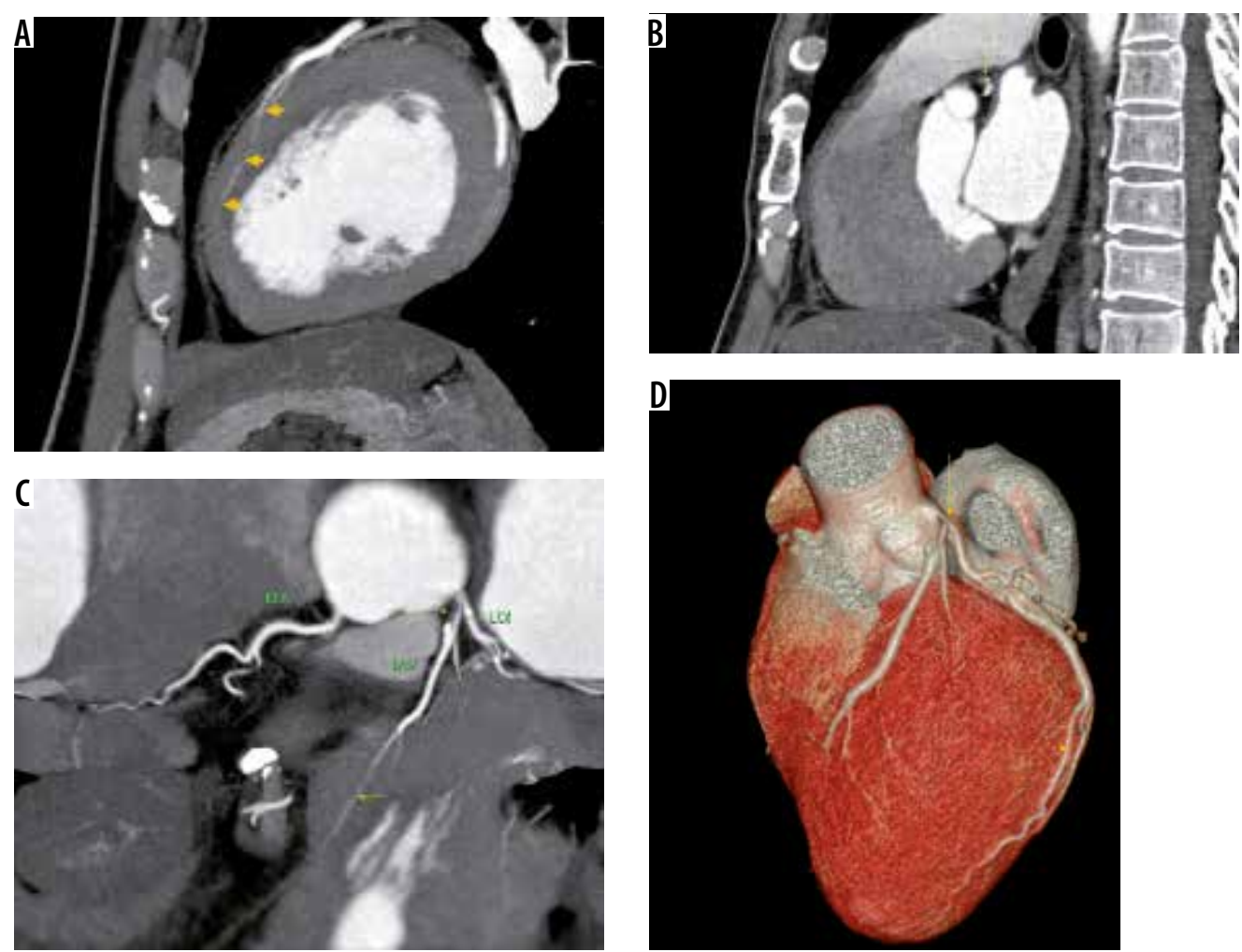

Figure 3. A) Sagittal reformat image showing myocardial bridging (arrows) of left anterior descending artery (LAD) involving a long segment. B) Sagittal reformat image showing coronary artery disease (arrow) in segment proximal to bridging in the same individual as in a. C) Reformat image showing myocardial bridging (short arrow) of LAD involving a long segment with coronary disease (long arrow) in the segment proximal to bridging in the same individual as in a. D) Volume rendered image showing myocardial bridging (short arrow) of LAD involving a long segment with coronary disease (long arrow) in the segment proximal to bridging in the same individual as in a 

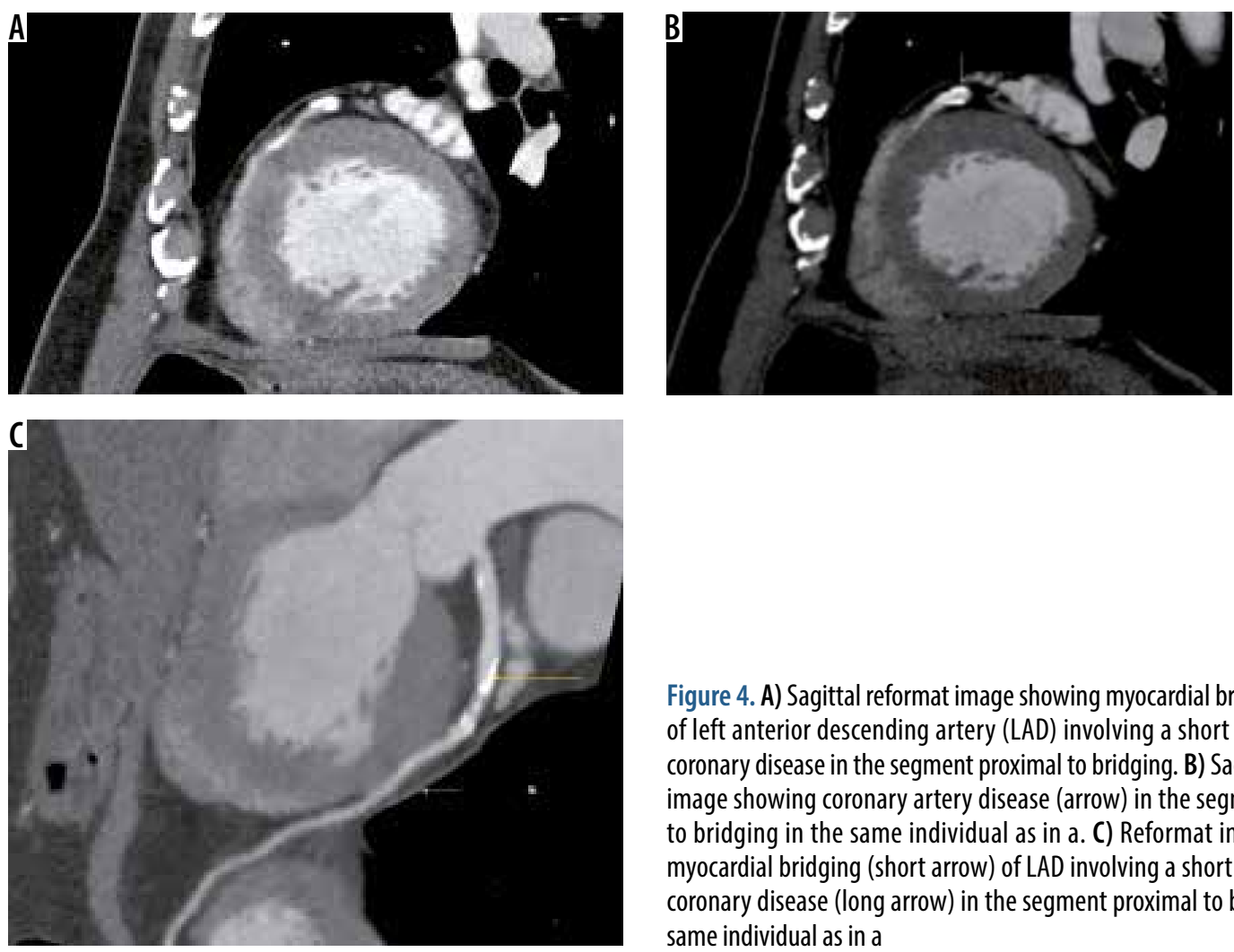

Figure 4. A) Sagittal reformat image showing myocardial bridging (arrow) of left anterior descending artery (LAD) involving a short segment with coronary disease in the segment proximal to bridging. B) Sagittal reformat image showing coronary artery disease (arrow) in the segment proximal to bridging in the same individual as in a. C) Reformat image showing myocardial bridging (short arrow) of LAD involving a short segment with coronary disease (long arrow) in the segment proximal to bridging in the same individual as in a

these two groups. However, in multivariate analysis, only age was significantly associated with proximal segment plaquing. Diabetes and the small length-to-depth ratio of the bridged segment became nonsignificant in multivariate analysis. In addition to the length and depth, this simple ratio (RA-MA ratio) may be an additional parameter in risk assessment of myocardial bridging. More complex parameters such as transluminal attenuation gradient are also emerging [24].

\section{Limitations}

The limitations of our study include its retrospective and case-control nature. Because the number of patients with bridging was small, the power of the study was lower. The degree of systolic deformation of vessels was not assessed in our study, which would be more correlative with the presence of a proximal segment plaque. Finally, a study cohort with chest pain syndromes will be different from the general population, limiting the extrapolation of this prevalence to the general population. The haemodynamics of blood flow in the tunnelled and proximal segment determined using intravascular Doppler ultrasound as a function of the length, depth, and their ratio on CTCA may provide insights into the anatomic grading of bridging and its physiological effects on the coronary plaque [25].

\section{Conclusions}

The MDCT prevalence of myocardial bridging in our population with chest pain syndromes was $10 \%$, with midLAD being the most common segment involved. Proximal segment plaquing was associated with age, presence of diabetes, and lower RA-MA ratio of the bridged segment in univariate analysis, but only age retained significance in multivariate analysis. We conclude that coronary plaques in LAD are similar in patients with and without myocardial bridging, and although a lower RA-MA ratio of the bridged segment influences proximal segment plaquing, it is not over and above what can be explained by patient age alone.

\section{Acknowledgements}

Special thanks to Dr. C.N. Manjunath for his guidance.

\section{Disclosure}

The authors declare no conflicts of interest. 


\section{References}

1. Reyman HC. Diss. de vasis cordis propriis. Bibl Anat 1737; 2: 359-379.

2. Portmann WC, Iwig J. Die intramurale koronarie im angiogramm. Fortschr Rontgenstr 1960; 92: 129-132.

3. Angelini P, Trivellato M, Donis J, Leachman RD. Myocardial bridges: a review. Prog Cardiovasc Dis 1983; 26: 75-88.

4. Mazzu A, Di Tano G, Cogode R, Lo Presti G. Myocardial bridging involving more than one site of the left anterior descending coronary artery: an uncommon cause of acute ischemic syndrome. Cathet Cardiovasc Diagn 1995; 34: 329-332.

5. Baldassarre S, Unger P, Renard M. Acute myocardial infarction and myocardial bridging: a case report. Acta Cardiol 1996; 51: 461-465.

6. Bauters C, Chmait A, Tricot O, Lamblin N, Van Belle E, Lablanche JM. Images in cardiovascular medicine: coronary thrombosis and myocardial bridging. Circulation 2002; 105: 130.

7. Yetman AT, McCrindle BW, MacDonald C, Freedom RM, Gow R. Myocardial bridging in children with hypertrophic cardiomyopathy - a risk factor for sudden death. N Engl J Med 2004; 339: 1201-1209.

8. Bestetti RB, Costa RS, Kazava DK, Oliveira JS. Can isolated myocardial bridging of the left anterior descending coronary artery be associated with sudden death during exercise? Acta Cardiol 1991; 46: 27-30.

9. Cheng TO. Myocardial bridging in a young patient with sudden death. Clin Cardiol 1997; 20: 743.

10. Hostiuc S, Rusu MC, Hostiuc M, Negoi RI, Negoi I. Cardiovascular consequences of myocardial bridging: A meta-analysis and metaregression. Sci Rep 2017; 7: 1-13.

11. Kantarci, Mecit, et al. Detection of myocardial bridging with ECG-gated MDCT and multiplanar reconstruction. Am J Roentgenol 2006; 186 (6 Suppl 2): S391-S394.

12. Rossi L, Dander B, Nidasio GP, et al. Myocardial bridges and ischemic heart disease. Eur Heart J 1980; 1: 239-245.

13. Geirenger E. The mural coronary. Am Heart J 1951; 41: 359-368.

14. Poláček P, Zechmeister A. The occurrence and significance of myocardial bridges and loops on coronary arteries. In: Krutna V (ed.). Monograph 36, Opuscola Cardiologica. Acta Facultatis Medicae Universitatis Brunenses. University J.E. Purkinje, Brno; 1968: 1-99.
15. Mohlenkamp S, Hort W, Ge J, Erbel R. Update on myocardial bridge. Circulation 2002; 106: 2616-2622.

16. Juillière $\mathrm{Y}$, Berder V, Suty-Selton $\mathrm{C}$, et al. Isolated myocardial bridges with angiographic milking of left anterior descending coronary artery: a long-term follow-up study. Am Heart J 1995; 129: 663-665.

17. Abuarqoub A, Naranjo M, Shamoon F. Myocardial bridging with left ventricular hypertrophy presenting as Wellens pattern. Ann Transl Med 2017; 5: 401.

18. Kawawa Y, Ishikawa Y, Gomi T, et al. Detection of myocardial bridge and evaluation of its anatomical properties by coronary multislice spiral computed tomography. Eur J Radiol 2007; 61: 130-138.

19. Leschka S, Koepfli P, Husmann L, et al. Myocardial bridging: depiction rate and morphology at CT coronary angiography: comparison with conventional coronary angiography. Radiology 2008; 246: 754-762.

20. Kim PJ, Hur G, Kim SY, et al. Frequency of myocardial bridges and dynamic compression of epicardial coronary arteries: a comparison between computed tomography and invasive coronary angiography. Circulation 2009; 119: 1408-1416.

21. Görmeli CA, Yagmur J, Özdemir R, et al. Comparison of myocardial bridging prevalence using 64-slice versus 256 -slice computed tomography scanners: what has changed with recent innovations in CT? Biomed Res 2016; 27: 954-958.

22. Liu G, Qu Y, Chen X, et al. Measurements of myocardial bridges on computed tomography predict presence of clinical symptoms and outcomes of adverse heart events: a retrospective study in a large population from China. Acta Radiol 2016; 58: 1068-1076.

23. Noble J, Bourassa MG, Petitclerc R, Dyrda I. Myocardial bridging and milking effect of the left anterior descending artery: normal variant or obstruction? Am J Cardiol 1976; 37: 993-999.

24. Yu M, Zhang Y, Li Y, et al. Assessment of myocardial bridge by cardiac CT: intracoronary transluminal attenuation gradient derived from diastolic phase predicts systolic compression. Korean J Radiol 2017; 18: 655-663.

25. Rogers IS, Tremmel JA, Schnittger I. Myocardial bridges: overview of diagnosis and management. Congenit Heart Dis 2017; 12: 619-623. 